\title{
$\gamma$-ray strength function for astrophysical applications in the IAEA-CRP
}

\author{
Hiroaki Utsunomiya ${ }^{1, *}$, Stephane Goriely ${ }^{2}$, Therese Renstrøm ${ }^{3}$, Gry M. Tveten ${ }^{3}$, Takashi Ari-izumi ${ }^{1}$, Shuji Miyamoto ${ }^{4}$, \\ Yiu-Wing Lui ${ }^{5}$, Ann-Cecilie Larsen ${ }^{3}$, Sunniva Siem ${ }^{3}$, Stephane Hilaire ${ }^{6}$, Sophie Péru ${ }^{6}$, and Arjan J. Koning ${ }^{7}$ \\ ${ }^{1}$ Department of Physics, Konan University, Kobe, Japan \\ ${ }^{2}$ Institut d'Astronomie et d'Astrophysique, Université Libre de Bruxelles, Belgium \\ ${ }^{3}$ Department of Physics, University of Oslo, Norway \\ ${ }^{4}$ Laboratory of Advanced Science and Technology for Industry, University of Hyogo, Japan \\ ${ }^{5}$ Cyclotron Institute, Texas A \& M university, USA \\ ${ }^{6} \mathrm{CEA}, \mathrm{DAM}, \mathrm{DIF}$, Arpajon, France \\ ${ }^{7}$ Nuclear Data Section, International Atomic Energy Agency, Vienna, Austria
}

\begin{abstract}
The $\gamma$-ray strength function $(\gamma \mathrm{SF})$ is a nuclear quantity that governs photoabsorption in $(\gamma, n)$ and photoemission in $(n, \gamma)$ reactions. Within the framework of the $\gamma$-ray strength function method, we use $(\gamma, n)$ cross sections as experimental constraints on the $\gamma \mathrm{SF}$ from the Hartree-Fock-Bogolyubov plus quasiparticlerandom phase approximation based on the Gogny D1M interaction for E1 and M1 components. The experimentally constrained $\gamma \mathrm{SF}$ is further supplemented with the zero-limit $M 1$ and $E 1$ strengths to construct the downward $\gamma \mathrm{SF}$ with which $(n, \gamma)$ cross sections are calculated. We investigate $(n, \gamma)$ cross sections in the context of astrophysical applications over the nickel and barium isotopic chains along the s-process path.
\end{abstract}

\section{$1 \gamma$-ray strength function}

The $\gamma$-ray strength function $(\gamma \mathrm{SF})[1-3]$ is a nuclear statistical quantity of describing the nuclear electromagnetic response that is employed in the Hauser-Feshbach (HF) model [4] of the compound nuclear reaction.

\section{1 downward $\gamma$-ray strength function}

The $\gamma \mathrm{SF}$ in the de-excitation mode which we refer to as downward $\gamma \mathrm{SF}$ is a key quantity in the HF model calculation of radiative neutron capture cross sections. The downward $\gamma \mathrm{SF}$ for dipole radiation with a given energy $\varepsilon_{\gamma}$ is defined $[1,5]$ by

$$
\overleftarrow{f_{X 1}}\left(\varepsilon_{\gamma}\right)=\frac{\left\langle\Gamma_{X 1}\left(\varepsilon_{\gamma}\right) / \varepsilon_{\gamma}^{3}\right\rangle}{D_{\ell}}
$$

Here $X$ is either electric $(E)$ or magnetic $(M), \Gamma_{X 1}\left(\varepsilon_{\gamma}\right)$ is a partial radiation width, the symbol \langle\rangle stands for unweighted averaging over included resonances, and $D_{\ell}$ is the average level spacing for s-wave $(\ell=0)$ or p-wave $(\ell=1)$ neutron resonances.

\section{2 upward $\gamma$-ray strength function}

In contrast, the $\gamma \mathrm{SF}$ in the excitation mode which we refer to as upward $\gamma \mathrm{SF}$ is defined $[1,5]$ by the average cross section for $E 1 / M 1$ photoabsorption $\left\langle\sigma_{X 1}\left(\varepsilon_{\gamma}\right)\right\rangle$ to the final states with all possible spins and parities [2]:

\footnotetext{
*e-mail: hiro@konan-u.ac.jp
}

$$
\overrightarrow{f_{X 1}}\left(\varepsilon_{\gamma}\right)=\frac{\varepsilon_{\gamma}^{-1}}{3(\pi \hbar c)^{2}}\left\langle\sigma_{X 1}\left(\varepsilon_{\gamma}\right)\right\rangle
$$

\section{Brink-Axel hypothesis $A, B$, and $C$}

It is convenient to define the Brink-Axel hypothesis $[6,7]$ in three versions $A, B$ and $C$ on the upward and downward $\gamma \mathrm{SF}$.

\subsection{Brink-Axel hypothesis A}

The version $\mathrm{A}$ is the equality of the upward $\gamma \mathrm{SF}$ built on the ground state and excited states. The photoabsorption cross section and thus the photoneutron cross section for GDR were assumed to be of Lorentzian shape. Historically this hypothesis has led to the experimental investigation of nuclear properties of hot nuclei $[8,9]$, which was triggered by the observation of radiations from GDR built on highly excited states [10].

\subsection{Brink-Axel hypothesis B}

The equality similar to the version A may apply to photodeexcitation as well. This version is backed by the detailed balance theorem [11] which links photo-emission and absorption between given initial and final states. Recently, it was experimentally shown that the equality of $\gamma \mathrm{SF}$ in photodeexcitation (downward $\gamma \mathrm{SF}$ ) from initial states at different excitation energies [12] and to different final states $\left(2^{+}\right.$and $\left.4^{+}\right)$holds under the presence of M1 upbend [13]. 


\subsection{Brink-Axel hypothesis C}

The version $\mathrm{C}$ is concerned with the equality of upward and downwad $\gamma$ SFs. A low-energy enhancement called M1 upbend was experimentally observed in downward $\gamma \mathrm{SF}$ [14-16] and theoretically supported by the shellmodel calculation [17-23]. A recent systematic study across the chart of nuclei has formulated the low-energy enhancement as zero-limit E1 and M1 strengths in the analytical form based on the shell-model calculation [24]. The presence of the zero-limit strength which corresponds to $\gamma$-ray transitions between high-lying states is unique to the downward $\gamma \mathrm{SF}$, showing that the Brink-Axel hypothesis $C$ is violated.

\section{Systematic study of $(\gamma, n)$ and $(n, \gamma)$ cross sections}

We present here a systematic investigation of the $(n, \gamma)$ and $(\gamma, n)$ cross section within the $\gamma$-ray strength function method $[25,26]$ in the context of astrophysical applications for $\mathrm{Ni}$ isotopes including ${ }^{63} \mathrm{Ni}$, a branching point nucleus along the weak s-process path and $\mathrm{Ba}$ isotopes in the vicinity of the neutron magic number 82 along the main s-process path.

\section{1 $\mathrm{Ni}$ isotopes}

Figure 1 shows downward $\gamma \mathrm{SFs}, \overleftarrow{f_{X 1}}\left(\varepsilon_{\gamma}\right)$, for Ni isotopes constructed in the present study [25]. The present experimental $(\gamma, n)$ cross sections for ${ }^{60} \mathrm{Ni},{ }^{61} \mathrm{Ni}$, and ${ }^{64} \mathrm{Ni}$ were used to constrain the $\gamma \mathrm{SF}$ from the HartreeFock-Bogolyubov plus quasiparticle-random phase approximation based on the Gogny D1M interaction for $E 1$ and $M 1$ components (hereafter denoted as D1M+QRPA). Phenomenological corrections include a broadening the QRPA strength to take the neglected damping of GDR into account and a shift of the strength to lower energies due to the contribution beyond one-particle -one-hole excitations and the coupling between the single-particle and low-lying collective phonon degrees of freedom (see Ref. [24, 25] for more details). The phenomenological correction was systematically applied throughout the $\mathrm{Ni}$ isotopic chain including ${ }^{59} \mathrm{Ni}$ and ${ }^{65} \mathrm{Ni}$. The Oslo data whenever available are shown in Fig. 1. We follow the same prescriptions as used in Ref. [24], i.e. the final $E 1$ and $M 1$ strengths, referred to as D1M+QRPA+0lim, include the QRPA as well as the zero-limit contributions and are expressed as

$$
\begin{aligned}
& \overleftarrow{f_{E 1}}\left(\varepsilon_{\gamma}\right)=f_{E 1}^{Q R P A}\left(\varepsilon_{\gamma}\right)+f_{0} U /\left[1+e^{\left(\varepsilon_{\gamma}-\varepsilon_{0}\right)}\right] \\
& \overleftarrow{f_{M 1}}\left(\varepsilon_{\gamma}\right)=f_{M 1}^{Q R P A}\left(\varepsilon_{\gamma}\right)+C e^{-\eta \varepsilon_{\gamma}}
\end{aligned}
$$

where an $M 1$ zero limit $C=10^{-8} \mathrm{MeV}^{-3}$ derived from shell-model calculations [24] was found to provide a rather good systematic description of available photoneutron data, average resonance capture data, Oslo $\gamma \mathrm{SF}$ as well as averaged radiative widths. Larger values could be envisioned from previous Oslo measurements [27, 29]. For this reason, two different values are adopted in the

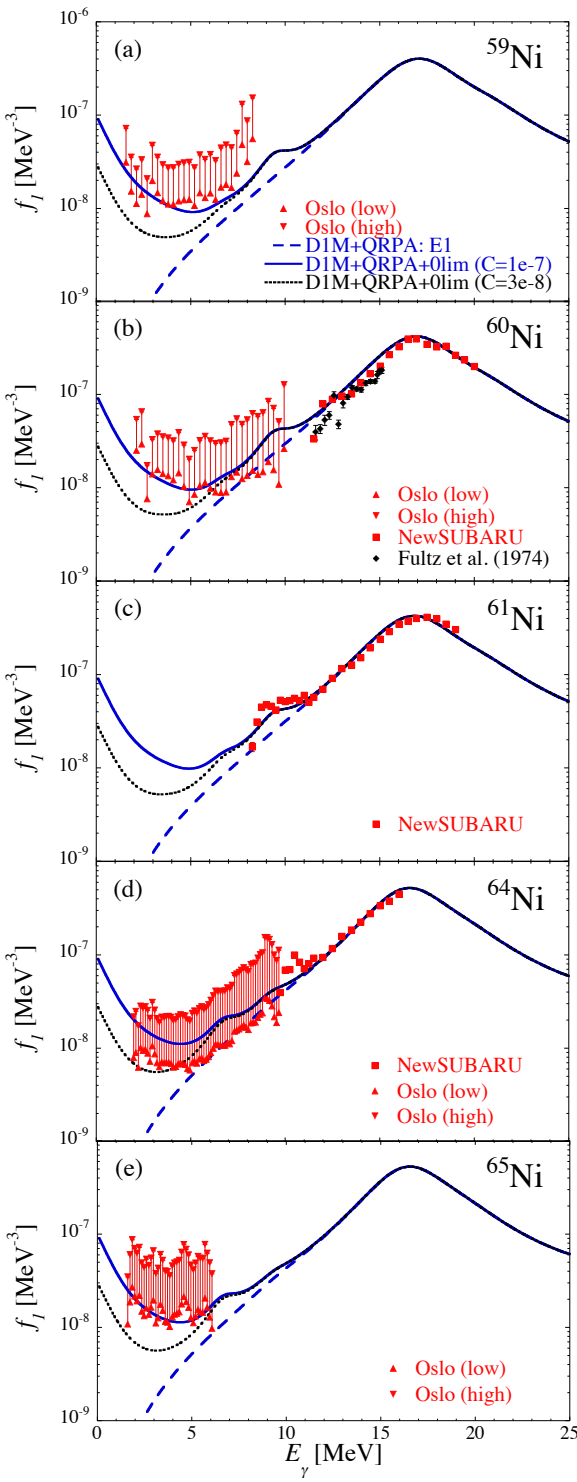

Figure 1. (Color online) (a-e) Downward $\gamma \mathrm{SFs}$ for the $59,60,61,64,65 \mathrm{Ni}$ isotopes. The red triangles correspond to the upper and lower limits of the $\gamma \mathrm{SF}$ extracted from the present Oslo data and the red squares to the present NewSUBARU photoneutron data. The dashed curve represents the D1M+QRPA $E 1$ strength and the dotted (full) line the D1M+QRPA+0lim $E 1+M 1$ dipole strength obtained with $C=3 \cdot 10^{-8} \mathrm{MeV}^{-3}\left(C=10^{-7} \mathrm{MeV}^{-3}\right)$. The $\gamma \mathrm{SF}$ of ${ }^{64,65} \mathrm{Ni}$ are taken from $[27,28]$. The $\gamma \mathrm{SF}$ extracted from the ${ }^{60} \mathrm{Ni}(\gamma, \mathrm{n})$ data of Fultz et al. [30] is also shown in panel (b).

present analysis, namely $C=3 \cdot 10^{-8}$ and $10^{-7} \mathrm{MeV}^{-3}$. The D1M+QRPA calculation is in relatively good agreement with the photoneutron data, even in the $10 \mathrm{MeV}$ region, where one can see extra M1 strength on top of the E1 component, as seen in ${ }^{61} \mathrm{Ni}$.

Figure 2 shows $(n, \gamma)$ cross sections predicted with the TALYS code [37] based on the downward $\gamma$ SF shown in Fig. 1 in comparison with experimental data. In addition to the $\gamma \mathrm{SF}$, the radiative neutron capture is rather sensitive to the nuclear level densities. For this reason, five 


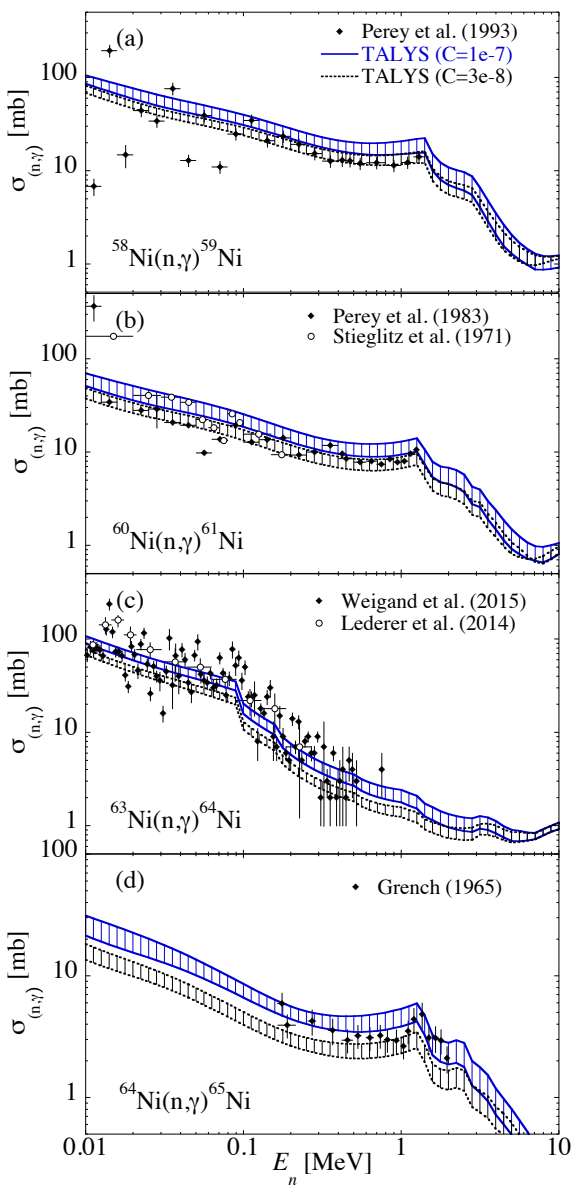

Figure 2. (Color online) radiative neutron capture cross section for the (a) ${ }^{58} \mathrm{Ni}$, (b) ${ }^{60} \mathrm{Ni}$, (c) ${ }^{63} \mathrm{Ni}$, and (d) ${ }^{64} \mathrm{Ni}$. The full (dotted) line corresponds to the TALYS calculation obtained with the D1M+QRPA+0lim dipole strength obtained with $C=$ $3 \cdot 10^{-8} \mathrm{MeV}^{-3}\left(C=10^{-7} \mathrm{MeV}^{-3}\right)$. Experimental data are taken from [31-36].

different nuclear level density models have been considered [38-41], all of them being adjusted to experimental low-lying states as well as s-wave resonance spacings whenever available experimentally [42]. The hashed areas shown in Fig. 2 represent the prediction uncertainties associated with different nuclear level density models. Radiative neutron capture cross sections are reasonably reproduced by the experimentally constrained downward $\gamma \mathrm{SF}$ with the zero-limit strength though it remains difficult to reconcile $\gamma \mathrm{SF}$ and cross section data in some cases.

\subsection{Ba isotopes}

Figure 3 shows upward $\gamma \mathrm{SFs}, \overrightarrow{f_{X 1}}\left(\varepsilon_{\gamma}\right)$, for ${ }^{137} \mathrm{Ba}$ and ${ }^{138} \mathrm{Ba}$ [47]. Two relatively different models of $\gamma \mathrm{SF}$, the semimicroscopic D1M+QRPA and phenomenological Simple Modified Lorentzian (SMLO) models, are employed. Similarly to $\mathrm{Ni}$ isotopes, the phenomenological correction is systematically applied to the $\mathrm{Ba}$ isotopic chain. In addition, a specific correction that is an energy shift of 0.5

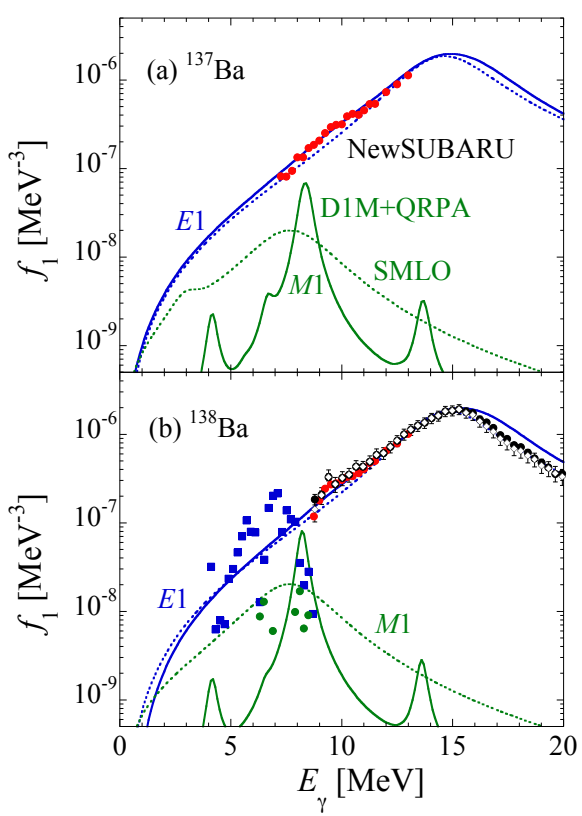

Figure 3. (Color online) (a) Comparison of the D1M+QRPA (solid lines) and SMLO (dotted lines) $\gamma \mathrm{SF}$ for ${ }^{137} \mathrm{Ba}$ with the measured strength function extracted from the present NewSUBARU experiment (red circles). The $E 1$ mode is shown by blue lines and $M 1$ by green lines. (b) same for and ${ }^{138} \mathrm{Ba} \gamma \mathrm{SF}$. Previous photoneutron data (solid circles) [44] and its evaluation (open diamonds) [45] are also shown. Nuclear Resonance fluorescence data [43] are shown by blue squares for the E1 strength and green circles for the M1 strength.

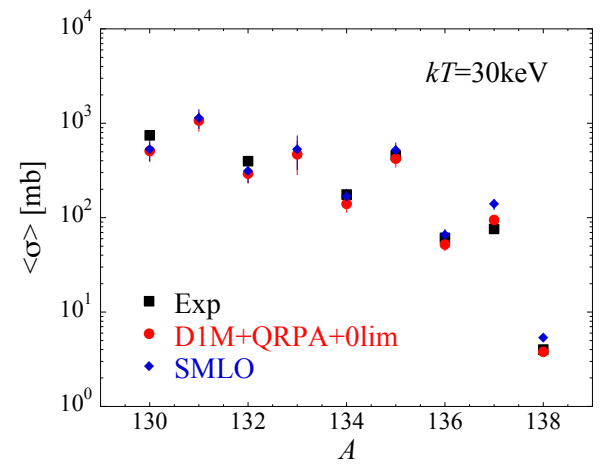

Figure 4. (Color online) Comparison between experimental (black squares) [48] and theoretical Maxwellian-averaged radiative neutron capture cross sections at $30 \mathrm{keV}$ predicted by the TALYS code for Ba isotopes with $A$ lying between 130 and 138 .

$\mathrm{MeV}$ of the overall $E 1$ strength, is required in the case of ${ }^{138} \mathrm{Ba}$.

Hauser-Feshbach model calculations of $(n, \gamma)$ cross sections and the Maxwellian-averaged cross sections (MACS) were performed with the TALYS code. The upward $\gamma \mathrm{SF}$ shown in Fig. 3 supplemented with the zerolimit $E 1$ and $M 1$ components was used as the downward $\gamma \mathrm{SF}$ for ${ }^{137} \mathrm{Ba}$ and ${ }^{138} \mathrm{Ba}$ in the TALYS calculation. Re- 
sults of a systematic study of the MACS over the Ba isotopic chain, including those for ${ }^{131} \mathrm{Ba}(\mathrm{n}, \gamma){ }^{132} \mathrm{Ba}$ and ${ }^{133} \mathrm{Ba}(\mathrm{n}, \gamma){ }^{134} \mathrm{Ba}$ reactions, are shown in Fig. 4 in comparison with experimental data [48].

\section{Summary}

There is a growing research interest in the study of the $\gamma$ ray strength function which governs photo-emission and absorption processes in nuclear physics and astrophysics. The Brink hypothesis in three versions has been a navigator of the experimental study of the $\gamma$-ray strength function in a variety of nuclear reactions such as radiative neutron capture, photoneutron, nuclear resonance fluorescence, inelastic and transfer reactions. We have systematically performed TALYS Hauser-Feshbach model calculation of $(n, \gamma)$ cross sections over the $\mathrm{Ni}$ and $\mathrm{Ba}$ isotopic chain along the s-process nucleosynthesis path based on the $\gamma$-ray strength function method, where $(\gamma, n)$ cross sections were used as experimental constraints on the upward D1M+QRPA $\gamma$-ray strength function and the downward $\gamma$-ray strength function was constructed by supplementing the upward $\gamma$-ray strength function with the zero-limit $M 1$ and $E 1$ strengths. The calculated $(n, \gamma)$ cross sections are in rather good agreement with experimental data.

\section{References}

[1] G.A. Bartholomew, E.D. Earle, A.J. Fergusson, J.W. Knowles, mad M.A. Lone, Adv. Nucl. Phys. 7, 229 (1973).

[2] M.A. Lone, Proc. 4th Int. Symp., Smolenice, Czechoslovakia, 1985, J. Kristin, E. Betak (eds.), D. Reidel, Dordrecht, Holland (1986) 238.

[3] S. Goriely et al., European Physical Journal A (2019), in press.

[4] W. Hauser and H. Feshbach, Phys. Rev. 87, 366 (1952).

[5] R. Capote et al., Nuclear Data Sheets 110, 3107 (2009).

[6] D.M. Brink, Ph.D thesis, Oxford University, 1955.

[7] P. Axel, Phys. Rev. 126, 671 (1962).

[8] D.M. Brink, Nucl. Phys. A 649 218c (1999).

[9] D.M. Brink, Talk presented at the Workshop on "Chaos and Collectivity in Many Body Systems" at the PMIPKS, Dresden, Germany, March 5-8 2008.

[10] J.O. Newton et al., Phys. Rev. Lett. 46, 1383 (1981).

[11] J. M. Blatt and V. F. Weisskopf, Theoretical Nuclear Physics, p. 530 (John Wiley \& Sons, Inc., New York, 1952).

[12] A.C. Larsen et al., J. Phys. G:Nucl. Part. Phys. 44, 064005 (2017).

[13] M.D. Jones et al., Phys. Rev. C 97, 024327 (2018).

[14] A. Voinov et al., Phys. Rev. Lett. 93, 142504 (2004).

[15] M. Guttormsen et al., Phys. Rev. C 71, 044307 (2005).

[16] E. Algin et al., Phys. Rev. C 78, 054321 (2008).

[17] R. Schwengner, S. Frauendorf, and A. C. Larsen, Phys. Rev. Lett. 111, 232504 (2013).
[18] B. A. Brown and A. C. Larsen, Phys. Rev. Lett. 113, 252502 (2014).

[19] K. Sieja, Phys. Rev. Lett. 119, 052502 (2017).

[20] K. Sieja, Europhys. J. Web Conf. 146, 05004 (2017).

[21] S. Karampagia, B. A. Brown, and V. Zelevinsky, Phys. Rev. C 95, 024322 (2017).

[22] R. Schwengner, S. Frauendorf, and B. A. Brown, Phys. Rev. Lett. 118, 092502 (2017).

[23] J. E. Midtbø, A. C. Larsen, T. Renstrøm, F. L. Bello Garrote, and E. Lima, Phys. Rev. C 98, 064321 (2018).

[24] S. Goriely, S. Hilaire, S. Péru, K. Sieja, Phys. Rev. C 98, 014327 (2018).

[25] H. Utsunomiya et al., Phy. Rev. C 98, 054619 (2018).

[26] H. Utsunomiya et al., Phy. Rev. C 99, 024609 (2019).

[27] L. Crespo Campo et al., Phys. Rev. C 94, 044321 (2016).

[28] L. Crespo Campo et al., Phys. Rev. C 96, 014312 (2017).

[29] E. Algin et al., Phys. Rev. C 78, 054321(2008).

[30] S.C. Fultz, R.A. Alvarez, B.L. Berman, and P. Meyer, Phys. Rev. C 10, 608 (1974).

[31] C.M. Perey, F.G. Perey, J.A. Harvey, N.W. Hill, N.M. Larson, R.L. Macklin, and D.C.Larson, Phys. Rev. C 47, 1143 (1993)

[32] C.M. Perey, J.A. Harvey, R.L. Macklin, F.G. Perey, and R.R. Winters, Phys. Rev. C 27, 2556 (1983)

[33] R.G. Stieglitz, R.W. Hockenbury, and R.C. Block, Nucl. Physics A 163, 592 (1971).

[34] M. Weigand, T.A. Bredeweg, A. Couture, et al., Phys. Rev. C 92, 045810 (2015)

[35] C. Lederer, C. Massimi, E. Berthoumieux, et al., Phys. Rev. C 89, 025810 (2014)

[36] H.A. Grench, Phys. Rev. B 140, 1277 (1965)

[37] A.J. Koning, D. Rochman, Nuclear Data Sheets 113, 2841 (2012).

[38] A.J. Koning, S. Hilaire, S. Goriely, Nucl. Phys. A 810, 13 (2008).

[39] P. Demetriou, S. Goriely, Nucl. Phys. A 695, 95 (2001).

[40] S. Goriely, S. Hilaire, and A.J. Koning, Phys. Rev. C 78, 064307 (2008).

[41] S. Hilaire, M. Girod, S. Goriely, and A.J. Koning, Phys. Rev. C 86, 064317 (2012).

[42] R. Capote, M. Herman, P. Oblozinsky, et al., Nuclear Data Sheets 110, 3107 (2009).

[43] A. P. Tonchev et al., Rev. Lett. 104, 072501 (2010).

[44] B.L. Berman, S.C. Fultz, J.T. Caldwell, M.A. Kelly, S.S. Dietrich, Phy. Rev. C 2, 2318 (1970).

[45] V.V. Varlamov, B.S. Ishkhanov, V.N. Orlin, N.N. Peskov, Yadernaya Fizika 79, 315 (2016).

[46] D.B. Stroud, D.M.H. Chan, Astrophys. J. 178, L93 (1972).

[47] H. Utsunomiya et al., Phys. Rev. C (2019), in press.

[48] Z.Y. Bao, H. Beer, F. Käppeler, F. Voss, K. Wisshak, T. Rauscher, At. Data Nucl. Data Tables 75, 1 (2000). 